J. Nonlinear Var. Anal. 3 (2019), No. 1, pp. 235-246

Available online at http://jnva.biemdas.com

https://doi.org/10.23952/jnva.3.2019.3.01

\title{
ATTRACTIVE POINTS OF FURTHER 2-GENERALIZED HYBRID MAPPINGS IN A COMPLETE CAT(0) SPACE
}

\author{
BASHIR ALI ${ }^{1, *}$, LAWAL YUSUF HARUNA ${ }^{2}$ \\ ${ }^{1}$ Department of Mathematical Sciences, Bayero University, Kano, Nigeria \\ ${ }^{2}$ Department of Mathematical Sciences, Kaduna State University, Kaduna, Nigeria
}

\begin{abstract}
In this paper, a new nonlinear mapping, which is said to be further 2-generalized hybrid, is introduced. The new mapping includes further generalized hybrid and normally 2-generalized hybrid mappings as special cases. A Halpern-type iterative scheme is constructed for attractive points of further 2-generalized hybrid mappings in a complete CAT( $(0) \operatorname{space}$.
\end{abstract}

Keywords. Attractive point; Further generalized hybrid mapping; Further 2-generalized hybrid mapping; Hadamard space; Normally 2-generalized hybrid mapping.

2010 Mathematics Subject Classification. 47H09, 47H10, 47J25.

\section{INTRODUCTION}

Let $C$ be a nonempty subset of a real Hilbert space $H$ and let $T: C \rightarrow H$ be a nonlinear mapping. We denote the sets of attractive and fixed points of $T$ by $A(T)$ and $F(T)$, respectively, i.e.,

$$
A(T)=\{u \in H:\|T v-u\| \leq\|v-u\|, \forall v \in C\}
$$

and

$$
F(T)=\{u \in C: T u=u\} .
$$

The concept of attractive points was first introduced in Hilbert space by Takahashi and Takeuchi [24]. The introduction was basically motivated to get rid of the closedness and convexity hypotheses imposed on nonempty subset $C \subset H$ in a celebrated Bailon's [2] nonlinear ergodic theorem. They established nonlinear ergodic theorem without convexity for generalized hybrid mappings in Hilbert spaces. Recall that a mapping $T: C \rightarrow H$ is said to be $(\alpha, \beta)$-generalized hybrid [14] if there exist $\alpha, \beta \in \mathbb{R}$ such that

$$
\alpha\|T x-T y\|^{2}+(1-\alpha)\|x-T y\|^{2} \leq \beta\|T x-y\|^{2}+(1-\beta)\|x-y\|^{2} \forall x, y \in C .
$$

Observe that mapping $T$ is reduced to a nonexpansive mapping, i.e.,

$$
\|T x-T y\| \leq\|x-y\|, \quad \forall x, y \in C
$$

if $\alpha=1$ and $\beta=0$. If $\alpha=\frac{3}{2}$ and $\beta=\frac{1}{2}$, then it is said to be hybrid [15, 23], i.e.,

$$
3\|T x-T y\|^{2} \leq\|x-y\|^{2}+\|T x-y\|^{2}+\|T y-x\|^{2}, \quad \forall x, y \in C .
$$

\footnotetext{
${ }^{*}$ Corresponding author.
}

E-mail addresses: bashiralik@yahoo.com (B. Ali), yulah121@gmail.com (L.H. Yusuf).

Received March 5, 2019; Accepted April 27, 2019.

(C)2019 Journal of Nonlinear and Variational Analysis 
Also, it is said to be nonspreading $[15,16]$, i.e.,

$$
2\|T x-T y\|^{2} \leq\|T x-y\|^{2}+\|T y-x\|^{2}, \quad \forall x, y \in C,
$$

if $\alpha=2$ and $\beta=1$. Recall that a mapping $T$ is said to be normally generalized hybrid [27] if there exist $\alpha, \beta, \gamma, \delta \in \mathbb{R}$ such that

(a) $\alpha+\beta+\gamma+\delta \geq 0$, (b) $\alpha+\beta>0$ or $\alpha+\gamma>0$ and

$$
\alpha\|T x-T y\|^{2}+\beta\|x-T y\|^{2}+\gamma\|T x-y\|^{2}+\delta\|x-y\|^{2} \leq 0, \quad \forall x, y \in C .
$$

As a generalization of the class of normally generalized hybrid mapping, the classes of normally 2generalized hybrid and further generalized hybrid mappings were introduced. Recall that a mapping $T$ is said to be

(i) normally 2-generalized hybrid [17] if there exist $\alpha_{1}, \alpha_{2}, \alpha_{3}, \beta_{1}, \beta_{2}, \beta_{3} \in \mathbb{R}$ such that (a) $\sum_{i=1}^{3}\left(\alpha_{i}+\right.$ $\left.\beta_{i}\right) \geq 0$ and (b) $\sum_{i=1}^{3} \alpha_{i}>0$ and

$$
\begin{aligned}
& \alpha_{1}\left\|T^{2} x-T y\right\|^{2}+\alpha_{2}\|T x-T y\|^{2}+\alpha_{3}\|x-T y\|^{2} \\
& \quad+\beta_{1}\left\|T^{2} x-y\right\|^{2}+\beta_{2}\|T x-y\|^{2}+\beta_{3}\|x-y\|^{2} \leq 0, \quad \forall x, y \in C .
\end{aligned}
$$

(ii) further generalized hybrid [22] if there exist $\alpha, \beta, \gamma, \delta, \varepsilon \in \mathbb{R}$ such that

(a) $\alpha+\beta+\gamma+\delta \geq 0, \varepsilon \geq 0$ (b) $\alpha+\beta>0$ or $\alpha+\gamma>0$ and

$$
\begin{aligned}
\alpha\|T x-T y\|^{2} & +\beta\|x-T y\|^{2}+\gamma\|T x-y\|^{2} \\
& +\delta\|x-y\|^{2}+\varepsilon\|x-T x\|^{2} \leq 0, \quad \forall x, y \in C .
\end{aligned}
$$

Weak and strong convergence theorems for attractive points of the above mentioned generalized nonlinear mappings have been studied in Hilbert spaces by various authors recently; see, for example, $[18,22,25,27]$ and the references contained therein. In 2018, Khan [22] proposed an iterative scheme that converges weakly to a common attractive point of two further generalized hybrid mappings in Hilbert spaces. For the strong convergence, Kondo and Takahashi [18] constructed a Halpern's type iterative scheme that converges strongly to a attractive point of normally 2-generalized hybrid mappings in Hilbert spaces without the condition that the domain of the mapping to be closed. They proved the following theorem.

Theorem 1.1. [18] Let $C$ be a nonempty and convex subset of $H$, and let $T: C \rightarrow C$ be a normally 2generalized hybrid mapping with $A(T) \neq \emptyset$. Let $\left\{\lambda_{n}\right\},\left\{a_{n}\right\},\left\{b_{n}\right\}$ and $\left\{c_{n}\right\}$ be sequences of real numbers in $(0,1)$ satisfying some conditions. Let $x_{1}, z \in C$ and define a sequence $\left\{x_{n}\right\}$ in $C$ as follows

$$
x_{n+1}=\lambda_{n} z+\left(1-\lambda_{n}\right)\left(a_{n} x_{n}+b_{n} T x_{n}+c_{n} T^{2} x_{n}\right)
$$

for all $n \in \mathbb{N}$. Then $x_{n} \rightarrow \bar{z}=P_{A(T)} z$.

Let $(X, d)$ be a metric space and $x, y \in X$. An isometry $c:[0, d(x, y)] \rightarrow X$ satisfying $c(0)=x$ and $c(d(x, y))=y$ is called a geodesic path joining $x$ to $y$. A geodesic segment between $x$ and $y$ is the image of a geodesic path joining $x$ to $y$, which is denoted by $[x, y]$ when it is unique. A geodesic space is a metric space $(X, d)$ in which every two points of $X$ are joined by a geodesic segment. It is said to be a uniquely geodesic space if every two points of $X$ are joined by only one geodesic segment. Let $X$ be a uniquely geodesic space and $(1-t) x \oplus t y$ denote the unique point $z$ of the geodesic segment joining $x$ to $y$ for each 
$x, y \in X$ such that $d(z, x)=t d(x, y)$ and $d(z, y)=(1-t) d(x, y)$. Set $[x, y]:=\{(1-t) x \oplus t y: t \in[0,1]\}$. Then a subset $C \subset X$ is said to be convex if $[x, y] \subset C$ for all $x, y \in C$.

Lemma 1.1. [3] Let $X$ be a uniquely geodesic space. The following are equivalent:

(i) $X$ is a $C A T(0)$ space.

(ii) $X$ satisfies the $C A T(0)(C N)$ inequality i.e. If $x, y, z$ are points in $X$ and $q$ is the midpoint of the segment $[y, z]$, then

$$
d^{2}(x, q) \leq \frac{1}{2} d^{2}(x, y)+\frac{1}{2} d^{2}(x, z)-\frac{1}{4} d^{2}(y, z)
$$

A complete CAT(0) space is called a Hadamard space. There are many Hadamard spaces such as Hilbert spaces, the Hilbert ball [9], Euclidean space $\mathbb{R}^{n}, \mathbb{R}$-trees, hyperbolic space [21] and any complete simply connected Riemanian manifold having non-positive sectional curvature. Let $\left\{x_{n}\right\}$ be a bounded sequence in a complete $\mathrm{CAT}(0)$ space $X$ and for $x \in X$,

$$
r\left(x,\left\{x_{n}\right\}\right):=\limsup _{n \rightarrow \infty} d\left(x, x_{n}\right) .
$$

The asymptotic radius of $\left\{x_{n}\right\}$ is given by

$$
r\left(\left\{x_{n}\right\}\right)=\inf \left\{r\left(x,\left\{x_{n}\right\}\right): x \in X\right\}
$$

and the asymptotic center of $\left\{x_{n}\right\}$ is the set

$$
A\left(\left\{x_{n}\right\}\right)=\left\{x \in X: r\left(x,\left\{x_{n}\right\}\right)=r\left(\left\{x_{n}\right\}\right)\right\} .
$$

In a complete CAT( 0$)$ sapce, it is known that $A\left(\left\{x_{n}\right\}\right)$ consists of exactly one point; see [7]. A sequence $\left\{x_{n}\right\}$ is said to be $\Delta$-convergent to a point $x \in X$ if for every subsequence $\left\{x_{n_{k}}\right\}$ of $\left\{x_{n}\right\}, A\left(\left\{x_{n_{k}}\right\}\right)=\{x\}$. In this case, $x$ is called the $\Delta$-limit of $\left\{x_{n}\right\}$ and it is written as $\Delta-\lim _{n \rightarrow \infty} x_{n}=x$.

The concept of the quasilinearisation in a complete CAT $(0)$ space was introduced by Berg and Nicolev [1]. They denoted a pair $(a, b) \in X \times X$ by $\overrightarrow{a b}$ and called it a vector. The quasilinearisation is a map $\langle.,\rangle:.(X \times X) \times(X \times X) \rightarrow \mathbb{R}$ defined by

$$
\langle\overrightarrow{a b}, \overrightarrow{c d}\rangle=\frac{1}{2}\left(d^{2}(a, d)+d^{2}(b, c)-d^{2}(a, c)-d^{2}(b, d)\right)
$$

for every $a, b, c, d \in X$. Following the definition, it is easy to see that, for all $a, b, c, d, e \in X$,

$$
\begin{gathered}
\langle\overrightarrow{a b}, \overrightarrow{a b}\rangle=d^{2}(a, b), \\
\langle\overrightarrow{b a}, \overrightarrow{c d}\rangle=-\langle\overrightarrow{a b}, \overrightarrow{c d}\rangle,
\end{gathered}
$$

and

$$
\langle\overrightarrow{a b}, \overrightarrow{c d}\rangle=\langle\overrightarrow{a e}, \overrightarrow{c d}\rangle+\langle\overrightarrow{e b}, \overrightarrow{c d}\rangle
$$

The space $X$ is said to satisfy the Cauchy Schwartz inequality if, for all $a, b, c, d \in X$,

$$
\langle\overrightarrow{a b}, \overrightarrow{c d}\rangle=d(a, b) d(c, d)
$$

It is known (see [1]) that a geodesically connected metric space is a CAT(0) space if and only if it satisfies the Cauchy-Schwartz inequality. 
The concept of dual space in a complete CAT( 0$)$ space $X$ was introduced by Kakavandi and Amini [12] is as follow: Let $C(X, \mathbb{R})$ be the space of all continuous real-valued functions on $X$. Consider a map $\Theta: \mathbb{R} \times X \times X \rightarrow C(X, \mathbb{R})$ defined by

$$
\Theta(t, a, b)(x)=t\langle\overrightarrow{a b}, \overrightarrow{a x}\rangle,(t \in \mathbb{R}, a, b, x \in X) .
$$

The Cauchy-Schwartz inequality implies $\Theta(t, a, b)$ is a Lipschitz function with Lipschitz semi-norm

$$
L(\Theta(t, a, b))=|t| d(a, b)(t \in \mathbb{R}, a, b \in X),
$$

where the Lipschitz semi-norm $L(\phi)$ of any function $\phi: X \rightarrow \mathbb{R}$ is given by

$$
L(\phi)=\sup \left\{\frac{\phi(x)-\phi(y)}{d(x, y)}: x, y \in X, x \neq y\right\}
$$

A pseudometric $D$ on $\mathbb{R} \times X \times X$ is defined by

$$
D((t, a, b),(s, c, d))=L(\Theta(t, a, b)-\Theta(s, c, d)),
$$

for $t, s \in \mathbb{R}$ and $a, b, c, d \in X$. In a complete CAT(0) space, it was shown [12] that $D((t, a, b),(s, c, d))=0$ if and only if

$$
t\langle\overrightarrow{a b}, \overrightarrow{x y}\rangle=s\langle\overrightarrow{c d}, \overrightarrow{x y}\rangle, \quad \forall x, y \in X
$$

Thus $D$ induces an equivalence relation on $\mathbb{R} \times X \times X$ with equivalence class defined by

$$
[t \overrightarrow{a b}]:=\{s \overrightarrow{c d}: D((t, a, b),(s, c, d))=0\}
$$

The pair $\left(X^{*}, D\right)$ is called the dual space of the metric space $(X, d)$, where

$$
X^{*}=\{[t \overrightarrow{a b}]:(t, a, b) \in \mathbb{R} \times X \times X\}
$$

and the function $D$ on $X^{*}$ is a metric.

The concept of attractive points of a nonlinear map $T$ was first studied in the setting of CAT(0) spaces by Kunwai, Kaewkhao and Inthakon [19]. They defined and denoted the set of attractive points $A(T)$ by

$$
A(T)=\{z \in X: d(z, T y) \leq d(z, y), \forall x \in C\}
$$

and established some of its properties. In 2015, Kaewkhao, Inthakon and Kunwai [10] proved the $\Delta$ convergence of a Mann-type scheme to a point in the set of attractive points of normally generalized hybrid mappings. Recently, Cuntavepanit and Phuengrattana [5] studied the class of further generalized hybrid mappings [22] in Hadamard spaces. They established the demiclosed principle and proved the $\Delta$-convergence for attractive points.

It is our purpose in this paper to introduce a further 2-generalized hybrid mapping, which includes normally 2-generalized hybrid and further generalized hybrid mappings as special cases, in a complete CAT(0) space. We then construct a Halpern's type iterative scheme to find an attractive point of such mappings. Our results improved and generalize many results in the literature. 


\section{PRELIMINARIES}

Throughout this paper, the symbols " $\rightarrow$ " and " $\rightarrow$ " represent the strong and the $\Delta$-convergence, respectively. The following results play vital roles in establishing our main result.

Lemma 2.1. [7] Let $X$ be a $C A T(0)$ space and $x, y \in X, t \in[0,1]$. Then

(i) $d(z, t x \oplus(1-t) y) \leq t d(z, x)+(1-t) d(z, y)$;

(ii) $d^{2}(z, t x \oplus(1-t) y) \leq t d^{2}(z, x)+(1-t) d^{2}(z, y)-t(1-t) d^{2}(x, y)$.

The following notation was introduced by Dompongsa, Kaewkhao and Panyanak [8] in CAT(0) spaces. Let $x_{1}, x_{2}, \ldots, x_{n}$ be points in $\operatorname{CAT}(0)$ spaces. For $\lambda_{1}, \lambda_{2}, \ldots, \lambda_{n} \in(0,1)$ with $\sum_{i=1}^{n} \lambda_{i}=1$, we write

$$
\oplus_{i=1}^{n} \lambda_{i} x_{i}=\left(1-\lambda_{n}\right)\left(\frac{\lambda_{1}}{1-\lambda_{n}} x_{1} \oplus \frac{\lambda_{2}}{1-\lambda_{n}} x_{2} \oplus \ldots \oplus \frac{\lambda_{n-1}}{1-\lambda_{n}} x_{n-1}\right) \oplus \lambda_{n} x_{n},
$$

where the definition of $\oplus$ is an ordered one in the sense that it depends on the order of points $x_{1}, \ldots, x_{n}$.

The following Lemma can be deduced from [4, Lemma 12]. For completeness, we give the shorter proof here.

Lemma 2.2. Let $X$ be a complete $C A T(0)$ space and $x, y \in X, t_{i} \in(0,1)$. Then

$$
d^{2}\left(z, \oplus_{i=0}^{n} t_{i} x_{i}\right) \leq \sum_{i=0}^{n} t_{i} d^{2}\left(z, x_{i}\right)-t_{j} t_{k} d^{2}\left(x_{j}, x_{k}\right)
$$

where $j, k \in\{0,1, \ldots, n\}$ and $\sum_{i=0}^{n} t_{i}=1$

Proof. Without loss of generality, we assume $j=0, k=1$ and apply induction on $n$. For $n=1$, the result follows from (ii) of Lemma 2.1. Suppose the result is true for $n=m$, for some $m \geq 1$, i.e.,

$$
d^{2}\left(z, \oplus_{i=0}^{m} t_{i} x_{i}\right) \leq \sum_{i=0}^{m} t_{i} d^{2}\left(z, x_{i}\right)-t_{0} t_{1} d^{2}\left(x_{0}, x_{1}\right)
$$

We now prove for $n=m+1$. By convexity of distance function in a complete CAT( 0$)$ space, we get

$$
\begin{aligned}
d^{2}\left(z, \oplus_{i=0}^{m+1} t_{i} x_{i}\right) & =d^{2}\left(z,\left(1-\alpha_{m+1}\right)\left(\frac{t_{1} x_{1}}{1-t_{m+1}} \oplus \ldots \oplus \frac{t_{m} x_{m}}{1-t_{m+1}}\right) \oplus t_{m+1} x_{m+1}\right) \\
& =d^{2}\left(z,\left(1-t_{m+1}\right) \oplus_{i=0}^{m} \frac{t_{i} x_{i}}{1-t_{m+1}} \oplus t_{m+1} x_{m+1}\right) \\
& \leq \sum_{i=0}^{m} t_{i} d^{2}\left(z, x_{i}\right)-t_{0} t_{1} d^{2}\left(x_{0}, x_{1}\right)+t_{m+1} d^{2}\left(z, x_{m+1}\right) \\
& =\sum_{i=0}^{m+1} t_{i} d^{2}\left(z, x_{i}\right)-t_{0} t_{1} d^{2}\left(x_{0}, x_{1}\right) .
\end{aligned}
$$

This completes the proof.

Lemma 2.3. [6] Let $X$ be a $C A T(0)$ space and $x, y, z \in X$. Then, for all $t \in[0,1]$,

$$
d^{2}(z, t x \oplus(1-t) y) \leq t^{2} d^{2}(z, x)+(1-t)^{2} d^{2}(z, y)-2 t(1-t)\langle\overrightarrow{x z}, \overrightarrow{y z}\rangle
$$

Lemma 2.4. [13] Let $X$ be a complete $C A T(0)$ space. Then every bounded sequence in $X$ has a $\Delta$ convergence subsequence. 
Kakavandi [11] introduced the notion of the $\mathbb{S}$ property for complete CAT( 0$)$ spaces and used it to characterize the concept of the $\Delta$-convergence in the space. A complete CAT(0)X is said to satisfy the $\mathbb{S}$ property if, for any $(x, y) \in X \times X$, there exists a point $y_{x} \in X$ such that $[\overrightarrow{x y}]=\left[\overrightarrow{y_{x} \vec{x}}\right]$.

Lemma 2.5. [11] Let $X$ be a complete CAT(0) space that satisfies the $\mathbb{S}$ property. Let $\left\{x_{n}\right\}$ be a bounded sequence in $X$ and $x \in X$. Then $\Delta-\lim _{n \rightarrow \infty} x_{n}=x$ if and only if $\lim _{n \rightarrow \infty}\left\langle\overrightarrow{x x_{n}}, \overrightarrow{x y}\right\rangle=0$ for all $y \in X$.

Lemma 2.6. [11] Let $X$ be a complete $C A T(0)$ space, $\left\{x_{n}\right\}$ a sequence in $X$ and $x \in X$. Then $\left\{x_{n}\right\}$ $\Delta$-converges to $x$ if and only if $\limsup _{n \rightarrow \infty}\left\langle\overrightarrow{x_{n} \vec{x}, \overrightarrow{y x}}\right\rangle \leq 0, \forall y \in X$.

Kaewkhao, Inthakon and Kunwai [10] proved that the set of attractive points of map $T$ is closed and convex in a complete $\mathrm{CAT}(0)$ space that satisfy $\left(\bar{Q}_{4}\right)$ condition. A complete $\mathrm{CAT}(0)$ space is said to satisfy $\left(\bar{Q}_{4}\right)$ condition [11] if, for any $x, y, p, q \in X, d(p, x)<d(x, q)$ and $d(p, y)<d(y, q)$ imply $d(p, m) \leq d(m, q), \forall m \in[x, y]$.

Lemma 2.7. [10] Let $(X, d)$ be a complete $C A T(0)$ space satisfying the $\left(\bar{Q}_{4}\right)$ condition. Let $C$ be a nonempty subset of $X$ and $T: C \rightarrow X$ be any map. Then, $A(T)$ is closed and convex.

Let $l^{\infty}$ be the Banach space of bounded sequences with supremum norm and let $\mu: l^{\infty} \rightarrow \mathbb{R}$ be a bounded and linear functional on $l^{\infty}$. Let $\mu(f)$ (or $\left.\mu_{n}\left(x_{n}\right)\right)$ denote the value of $\mu$ at $f=\left(x_{1}, x_{2}, x_{3}, \ldots ..\right) \in$ $l^{\infty}$. A mean $\mu_{n}$ is a linear functional defined on $l^{\infty}$ satisfying $\mu(e)=\|\mu\|=1$, where $e=(1,1,1, \ldots)$. And a Banach limit on $l^{\infty}$ is a mean $\mu_{n}$ such that $\mu_{n}\left(x_{n+1}\right)=\mu_{n}\left(x_{n}\right)$. The existence a Banach limit on $l^{\infty}$ is well known. In fact, if $\mu$ is a Banach limit on $l^{\infty}$, then, for $f=\left(x_{1}, x_{2}, x_{3}, \ldots ..\right) \in l^{\infty}$,

$$
\liminf _{n \rightarrow \infty} x_{n} \leq \mu_{n}\left(x_{n}\right) \leq \limsup _{n \rightarrow \infty} x_{n} .
$$

In particular, if $f=\left(x_{1}, x_{2}, x_{3}, \ldots ..\right) \in l^{\infty}$ and $x_{n} \rightarrow l \in \mathbb{R}$, then $\mu(f)=\mu_{n}\left(x_{n}\right)=l$. For the proof of the existence of a Banach limit and its elementary properties; see [26].

Lemma 2.8. $[10,19]$ Let $C$ be a nonempty subset of a complete $C A T(0)$ space $X$ and $T: C \rightarrow C$ be a mapping. Let $\left\{x_{n}\right\}$ be a bounded sequence in $C$ such that $\lim _{n \rightarrow \infty} d\left(x_{n}, T x_{n}\right)=0$. Then

(i) the sequences $\left\{d\left(x_{n}, y\right)\right\}$ and $\left\{d\left(T x_{n}, y\right)\right\}$ are bounded for all $y \in C$;

(ii) $\mu_{n} d\left(x_{n}, y\right)=\mu d\left(T x_{n}, y\right)$ for any Banach limit $\mu_{n}$ on $l^{\infty}$.

Lemma 2.9. [28] Let $\left\{a_{n}\right\}$ be a sequence of nonnegative real numbers satisfying the following relation:

$$
a_{n+1} \leq\left(1-\alpha_{n}\right) a_{n}+\alpha_{n} \delta_{n}, n \geq n_{0},
$$

where $\left\{\alpha_{n}\right\} \subset(0,1)$ and $\left\{\delta_{n}\right\}$ is a real sequence satisfying the following conditions $\lim _{n \rightarrow \infty} \alpha_{n}=0$, $\sum_{n=1}^{\infty} \alpha_{n}=\infty$ and $\limsup _{n \rightarrow \infty} \delta_{n} \leq 0$. Then $\lim _{n \rightarrow \infty} a_{n}=0$.

Lemma 2.10. [20] Let $\left\{a_{n}\right\}$ be a sequence of real numbers such that there exists a subsequence $\left\{n_{i}\right\}$ of $\{n\}$ such that $a_{n_{i}} \leq a_{n_{i}+1}$ for all $i \in \mathbb{N}$. Then there exists a non-decreasing sequence $\left\{m_{k}\right\} \subset \mathbb{N}$ such that $m_{k} \rightarrow \infty$ and the following properties are satisfied by all (sufficiently large) numbers $k \in \mathbb{N}$ :

$$
a_{m_{k}} \leq a_{m_{k}+1}
$$

and

$$
a_{k} \leq a_{m_{k}+1}
$$

In fact, $m_{k}=\max \left\{j \leq k: a_{j}<a_{j+1}\right\}$. 


\section{Main Results}

In this section, $X$ is considered to be a complete CAT( 0$)$ space. We start by introducing a new generalized nonlinear mapping called further 2-generalized hybrid mapping in Hadamard spaces. We then construct a Halpern type iterative scheme that converges strongly to a attractive point of a further 2generalized hybrid mapping.

Definition 3.1. Let $X$ be a complete CAT(0) space and let $C$ be a nonempty subset of $X$. A mapping $T: C \rightarrow C$ is said to be further 2-generalized hybrid if there exist $\alpha_{1}, \alpha_{2}, \alpha_{3}, \beta_{1}, \beta_{2}, \beta_{3}, \varepsilon_{1}, \varepsilon_{2} \in \mathbb{R}$ such that (i) $\sum_{i=1}^{3}\left(\alpha_{i}+\beta_{i}\right) \geq 0, \varepsilon_{1}, \varepsilon_{2} \geq 0$, (ii) $\sum_{i=1}^{3} \alpha_{i}>0$ and

$$
\begin{aligned}
\text { (iii) } \alpha_{1} \mathrm{~d}^{2}\left(\mathrm{~T}^{2} \mathrm{x}, \mathrm{Ty}\right)+\alpha_{2} \mathrm{~d}^{2}(\mathrm{Tx}, \mathrm{Ty})+\alpha_{3} \mathrm{~d}^{2}(\mathrm{x}, \mathrm{Ty})+\beta_{1} \mathrm{~d}^{2}\left(\mathrm{~T}^{2} \mathrm{x}, \mathrm{y}\right) \\
+\beta_{2} d^{2}(T x, y)+\beta_{3} d^{2}(x, y)+\varepsilon_{1} d^{2}\left(x, T^{2} x\right)+\varepsilon_{2} d^{2}(x, T x) \leq 0 .
\end{aligned}
$$

for all $x, y \in C$.

Remark 3.1. If $\alpha_{1}=\beta_{1}=\varepsilon_{2}=0$, then the mapping is reduced to a further generalized hybrid mapping in the sense of [5]. Also, the mapping is reduced to a normally 2-generalized hybrid mapping if $\varepsilon_{1}=\varepsilon_{2}=0$, i.e., there exist $\alpha_{1}, \alpha_{2}, \alpha_{3}, \beta_{1}, \beta_{2}, \beta_{3} \in \mathbb{R}$ such that (i) $\sum_{i=1}^{3}\left(\alpha_{i}+\beta_{i}\right) \geq 0$, (ii) $\sum_{i=1}^{3} \alpha_{i}>0$ and

$$
\text { (iii) } \begin{aligned}
\alpha_{1} \mathrm{~d}^{2}\left(\mathrm{~T}^{2} \mathrm{x}, \mathrm{Ty}\right) & +\alpha_{2} d^{2}(T x, T y)+\alpha_{3} d^{2}(x, T y)+\beta_{1} d^{2}\left(T^{2} x, y\right) \\
& +\beta_{2} d^{2}(T x, y)+\beta_{3} d^{2}(x, y) \leq 0 .
\end{aligned}
$$

for all $x, y \in C$.

Lemma 3.1. Let $C$ be a nonempty subset of a complete $C A T(0)$ space $X$ which satisfy the $(\mathbb{S})$ property and let $T: C \rightarrow C$ be a further 2-generalized hybrid mapping. Let $\left\{x_{n}\right\}$ be a bounded sequence in $C$ such that $\Delta-\lim _{n \rightarrow \infty} x_{n}=z$ and $d\left(x_{n}, T x_{n}\right) \rightarrow 0, d\left(x_{n}, T^{2} x_{n}\right) \rightarrow 0$ as $n \rightarrow \infty$. Then $z \in A(T)$.

Proof. Since $\left\{x_{n}\right\}$ is bounded, one sees from Lemma 2.7 that $\left\{d\left(x_{n}, y\right)\right\},\left\{d\left(T x_{n}, y\right)\right\}$ and $\left\{d\left(T^{2} x_{n}, y\right)\right\}$ are bounded. Now replacing $x$ with $x_{n}$ in (iii) of Definition 3.1, we get

$$
\begin{aligned}
& \alpha_{1} d^{2}\left(T^{2} x_{n}, T y\right)+\alpha_{2} d^{2}\left(T x_{n}, T y\right)+\alpha_{3} d^{2}\left(x_{n}, T y\right)+\beta_{1} d^{2}\left(T^{2} x_{n}, y\right) \\
& \quad+\beta_{2} d^{2}\left(T x_{n}, y\right)+\beta_{3} d^{2}\left(x_{n}, y\right)+\varepsilon_{1} d^{2}\left(x_{n}, T^{2} x_{n}\right)+\varepsilon_{2} d^{2}\left(x_{n}, T x_{n}\right) \leq 0 .
\end{aligned}
$$

for all $y \in C$. By applying Banach limit on both sides of the above inequality and the boundedness of $\left\{x_{n}\right\}$, we get

$$
\begin{aligned}
& \alpha_{1} \mu_{n} d^{2}\left(T^{2} x_{n}, T y\right)+\alpha_{2} \mu_{n} d^{2}\left(T x_{n}, T y\right)+\alpha_{3} \mu_{n} d^{2}\left(x_{n}, T y\right)+\beta_{1} \mu_{n} d^{2}\left(T^{2} x_{n}, y\right) \\
& \quad+\beta_{2} \mu_{n} d^{2}\left(T x_{n}, y\right)+\beta_{3} \mu_{n} d^{2}\left(x_{n}, y\right)+\varepsilon_{1} \mu_{n} d^{2}\left(x_{n}, T^{2} x_{n}\right)+\varepsilon_{2} \mu_{n} d^{2}\left(x_{n}, T x_{n}\right) \leq 0 .
\end{aligned}
$$

for all $y \in C$. This implies

$$
\left(\alpha_{1}+\alpha_{2}+\alpha_{3}\right) \mu_{n} d^{2}\left(x_{n}, T y\right)+\left(\beta_{1}+\beta_{2}+\beta_{3}\right) \mu_{n} d^{2}\left(x_{n}, y\right) \leq 0,
$$

for all $y \in C$. Since $\sum_{i=1}^{3} \alpha_{i}>0$, we obtain from definition 3.1 that

$$
\mu_{n} d^{2}\left(x_{n}, T y\right) \leq-\frac{\left(\beta_{1}+\beta_{2}+\beta_{3}\right)}{\left(\alpha_{1}+\alpha_{2}+\alpha_{3}\right)} \mu_{n} d^{2}\left(x_{n}, y\right),
$$

for all $y \in C$. Using the fact that $\sum_{i=1}^{3}\left(\alpha_{i}+\beta_{i}\right) \geq 0$, we get

$$
\mu_{n} d^{2}\left(x_{n}, T y\right) \leq \mu_{n} d^{2}\left(x_{n}, y\right)
$$


for all $y \in C$. Since $X$ satisfy $\mathbb{S}$ property and $\Delta-\lim _{n \rightarrow \infty} x_{n}=z$, we find from Lemma 2.5 that $\lim _{n \rightarrow \infty}$ $\left\langle\overrightarrow{z x_{n}}, \overrightarrow{z y}\right\rangle=0$ for all $y \in C$. By definition of quasilinearization, we get

$$
\lim _{n \rightarrow \infty}\left(d^{2}\left(x_{n}, z\right)-d^{2}\left(x_{n}, y\right)+d^{2}(z, y)\right)=0,
$$

for all $y \in C$. Thus

$$
\mu\left(d^{2}\left(x_{n}, z\right)-d^{2}\left(x_{n}, y\right)+d^{2}(z, y)\right)=0 .
$$

From (3.1), we get

$$
-\mu_{n} d^{2}\left(x_{n}, y\right) \leq-\mu_{n} d^{2}\left(x_{n}, T y\right)
$$

This implies

$$
\begin{aligned}
-\mu_{n} d^{2}\left(x_{n}, y\right) & +\mu\left(d^{2}\left(x_{n}, z\right)+d^{2}(z, y)+d^{2}(z, T y)\right) \\
& \leq-\mu_{n} d^{2}\left(x_{n}, T y\right)+\mu\left(d^{2}\left(x_{n}, z\right)+d^{2}(z, y)+d^{2}(z, T y)\right) .
\end{aligned}
$$

By rearranging, we get

$$
\begin{aligned}
d^{2}(z, T y) & +\mu\left(d^{2}\left(x_{n}, z\right)-d^{2}\left(x_{n}, y\right)+d^{2}(z, y)\right) \\
& \leq d^{2}(z, y)+\mu\left(d^{2}\left(x_{n}, z\right)-d^{2}\left(x_{n}, T y\right)+d^{2}(z, T y)\right) .
\end{aligned}
$$

Thus, $d(z, T y) \leq d(z, y)$. Hence $z \in A(T)$. This completes the proof.

Theorem 3.1. Let $C$ be a nonempty, convex subset of a complete $C A T(0)$ space $X$ which satisfy property $(\mathbb{S})$ and condition $\left(\bar{Q}_{4}\right)$. Let $T: C \rightarrow C$ be a further 2-generalized hybrid mapping such that $A(T) \neq \emptyset$. Let $\left\{x_{n}\right\}$ be a sequence generated by $u, x_{1} \in C$

$$
\left\{\begin{array}{l}
y_{n}=\alpha_{n} x_{n} \oplus \beta_{n} T x_{n} \oplus \gamma_{n} T^{2} x_{n} \\
x_{n+1}=\delta_{n} u \oplus\left(1-\delta_{n}\right) y_{n},
\end{array} .\right.
$$

where $\left\{\alpha_{n}\right\},\left\{\beta_{n}\right\},\left\{\gamma_{n}\right\}$ and $\left\{\delta_{n}\right\}$ are real sequences satisfying $\left\{\alpha_{n}\right\},\left\{\beta_{n}\right\},\left\{\gamma_{n}\right\} \subset[a, b] \subset(0,1)$ with $\alpha_{n}+\beta_{n}+\gamma_{n}=1$ and $\left(C_{1}\right): \lim _{n \rightarrow \infty} \delta_{n}=0,\left(C_{2}\right): \sum_{n=1}^{\infty} \delta_{n}=+\infty$. Then $\left\{x_{n}\right\}$ converges strongly to $z=$ $P_{A(T)}(u)$.

Proof. From Lemma 2.7, we see that $A(T)$ is closed and convex. So, $z=\mathrm{P}_{A(T)}(u)$ is well defined. Let $z=\mathrm{P}_{A(T)}(u) \in A(T)$. From equations (3.2), Lemma 2.1 and definition of $A(T)$, we have

$$
\begin{aligned}
d\left(z, y_{n}\right) & =d\left(z, \alpha_{n} x_{n} \oplus \beta_{n} T x_{n} \oplus \gamma_{n} T^{2} x_{n}\right) \\
& \leq \alpha_{n} d\left(z, x_{n}\right)+\beta_{n} d\left(z, T x_{n}\right)+\gamma_{n} d\left(z, T^{2} x_{n}\right) \\
& \leq \alpha_{n} d\left(z, x_{n}\right)+\beta_{n} d\left(z, x_{n}\right)+\gamma_{n} d\left(z, x_{n}\right) . \\
& =d\left(z, x_{n}\right) .
\end{aligned}
$$

Similarly,

$$
\begin{aligned}
d\left(z, x_{n+1}\right) & =d\left(z, \delta_{n} u \oplus\left(1-\delta_{n}\right) y_{n}\right) \\
& =\delta_{n} d(z, u)+\left(1-\delta_{n}\right) d\left(z, y_{n}\right) \\
& \leq \delta_{n} d(z, u)+\left(1-\delta_{n}\right) d\left(z, x_{n}\right) .
\end{aligned}
$$

By induction, we obtain

$$
d\left(z, x_{n+1}\right) \leq \max \left\{d(z, u), d\left(z, x_{n}\right)\right\}, \quad \forall n \geq 1,
$$


which implies that $\left\{d\left(z, x_{n}\right)\right\},\left\{x_{n}\right\},\left\{T x_{n}\right\}$ and $\left\{T^{2} x_{n}\right\}$ are bounded. By (3.2) and Lemma 2.2, we have

$$
\begin{aligned}
d^{2}\left(z, y_{n}\right) & =d^{2}\left(z, \alpha_{n} x_{n} \oplus \beta_{n} T x_{n} \oplus \gamma_{n} T^{2} x_{n}\right) \\
& \leq \alpha_{n} d^{2}\left(z, x_{n}\right)+\beta_{n} d^{2}\left(z, T x_{n}\right)+\gamma_{n} d^{2}\left(z, T^{2} x_{n}\right)-\alpha_{n} \beta_{n} d^{2}\left(x_{n}, T x_{n}\right) \\
& \leq \alpha_{n} d^{2}\left(z, x_{n}\right)+\beta_{n} d^{2}\left(z, x_{n}\right)+\gamma_{n} d^{2}\left(z, x_{n}\right)-\alpha_{n} \beta_{n} d^{2}\left(x_{n}, T x_{n}\right) \\
& =d^{2}\left(z, x_{n}\right)-\alpha_{n} \beta_{n} d^{2}\left(x_{n}, T x_{n}\right) .
\end{aligned}
$$

Thus,

$$
d^{2}\left(z, y_{n}\right) \leq d^{2}\left(z, x_{n}\right)-\alpha_{n} \beta_{n} d^{2}\left(x_{n}, T x_{n}\right)
$$

Similarly,

$$
d^{2}\left(z, y_{n}\right) \leq d^{2}\left(z, x_{n}\right)-\alpha_{n} \gamma_{n} d^{2}\left(x_{n}, T^{2} x_{n}\right)
$$

It follows from (3.2), Lemma 2.1, (3.4) and (3.5) that

$$
\begin{aligned}
d^{2}\left(z, x_{n+1}\right) & =d^{2}\left(z, \delta_{n} u \oplus\left(1-\delta_{n}\right) y_{n}\right) \\
& \leq \delta_{n} d^{2}(z, u)+\left(1-\delta_{n}\right) d^{2}\left(z, y_{n}\right) \\
& \leq \delta_{n} d^{2}(z, u)+\left(1-\delta_{n}\right) d^{2}\left(z, x_{n}\right) \\
& -\left(1-\delta_{n}\right) \alpha_{n} \beta_{n} d^{2}\left(x_{n}, T x_{n}\right) \\
& =\delta_{n}\left[d^{2}(z, u)-d^{2}\left(z, x_{n}\right)+\alpha_{n} \beta_{n} d^{2}\left(x_{n}, T x_{n}\right)\right] \\
& +d^{2}\left(z, x_{n}\right)-\alpha_{n} \beta_{n} d^{2}\left(x_{n}, T x_{n}\right) .
\end{aligned}
$$

Putting

$$
k_{1}=\sup \left\{\left|d^{2}(z, u)-d^{2}\left(z, x_{n}\right)\right|+\alpha_{n} \beta_{n} d^{2}\left(x_{n}, T x_{n}\right)\right\}
$$

and

$$
k_{2}=\sup \left\{\left|d^{2}(z, u)-d^{2}\left(z, x_{n}\right)\right|+\alpha_{n} \gamma_{n} d^{2}\left(x_{n}, T^{2} x_{n}\right)\right\},
$$

we get

$$
d^{2}\left(z, x_{n+1}\right) \leq d^{2}\left(z, x_{n}\right)-\alpha_{n} \beta_{n} d^{2}\left(x_{n}, T x_{n}\right)+\delta_{n} k_{1},
$$

and

$$
d^{2}\left(z, x_{n+1}\right) \leq d^{2}\left(z, x_{n}\right)-\alpha_{n} \gamma_{n} d^{2}\left(x_{n}, T^{2} x_{n}\right)+\delta_{n} k_{2} .
$$

These imply

$$
\alpha_{n} \beta_{n} d^{2}\left(x_{n}, T x_{n}\right) \leq d^{2}\left(z, x_{n}\right)-d^{2}\left(z, x_{n+1}\right)+\delta_{n} k_{1},
$$

and

$$
\alpha_{n} \gamma_{n} d^{2}\left(x_{n}, T^{2} x_{n}\right) \leq d^{2}\left(z, x_{n}\right)-d^{2}\left(z, x_{n+1}\right)+\delta_{n} k_{2} .
$$

We next consider the following cases.

Case 1. Assume sequence $\left\{d\left(z, x_{n}\right)\right\}$ is non-increasing. Since it is bounded, it is convergent. Thus, we have that

$$
d^{2}\left(z, x_{n}\right)-d^{2}\left(z, x_{n+1}\right) \rightarrow 0 \text { as } n \rightarrow \infty .
$$

Using $C_{1}$, equations (3.6), (3.7) and (3.8) we have $\alpha_{n} \beta_{n} d^{2}\left(x_{n}, T x_{n}\right) \rightarrow 0$ and $\alpha_{n} \gamma_{n} d^{2}\left(x_{n}, T^{2} x_{n}\right) \rightarrow 0$ as $n \rightarrow \infty$. Using the fact that $\alpha_{n}, \beta_{n}, \gamma_{n} \in[a, b] \subset(0,1)$, we obtain

$$
\lim _{n \rightarrow \infty} d\left(x_{n}, T x_{n}\right)=0, \quad \lim _{n \rightarrow \infty} d\left(x_{n}, T^{2} x_{n}\right)=0 .
$$


From (3.2) and (3.9) we get

$$
\begin{aligned}
d\left(x_{n}, y_{n}\right) & \leq \alpha_{n} d\left(x_{n}, x_{n}\right)+\beta_{n} d\left(x_{n}, T x_{n}\right) \\
& +\gamma_{n} d^{2}\left(x_{n}, T^{2} x_{n}\right) \rightarrow 0 \text { as } n \rightarrow \infty
\end{aligned}
$$

Using (3.2) and $C 1$, we get

$$
\begin{aligned}
d\left(y_{n}, x_{n+1}\right) & =d\left(y_{n}, \delta_{n} u \oplus\left(1-\delta_{n}\right) y_{n}\right) \\
& \leq \delta_{n} d\left(y_{n}, u\right)+\left(1-\delta_{n}\right) d\left(y_{n}, y_{n}\right) \\
& =\delta_{n} d\left(y_{n}, u\right) \rightarrow 0 \text { as } n \rightarrow \infty .
\end{aligned}
$$

It follows from (3.10) and (3.11) that

$$
\lim _{n \rightarrow \infty} d\left(x_{n}, x_{n+1}\right)=0 .
$$

Since $X$ is a complete CAT(0) space and $\left\{x_{n}\right\}$ is bounded, one obtains from Lemma 2.4 that there exists a subsequence $\left\{x_{n_{k}}\right\}$ of $\left\{x_{n}\right\}$ such that $\Delta-\lim _{k \rightarrow \infty} x_{n_{k}}=z$. From $\lim _{k \rightarrow \infty} d\left(x_{n_{k}}, T x_{n_{k}}\right)=0$ and $\lim _{k \rightarrow \infty} d\left(x_{n_{k}}, T^{2} x_{n_{k}}\right)=0$, we obtain from Lemma 3.1 that $z \in A(T)$. Using Lemma 2.6, we have

$$
\limsup _{n \rightarrow \infty}\left\langle\overrightarrow{u z}, \overrightarrow{x_{n} z}\right\rangle=\limsup _{k \rightarrow \infty}\left\langle\overrightarrow{u z}, \overrightarrow{x_{n_{k}}} \vec{z}\right\rangle \leq 0,
$$

for all $u \in X$. Using the quasilinearization property, the Cauchy-Swartz inequality, (3.10) and (3.13), we have that

$$
\begin{aligned}
\limsup _{n \rightarrow \infty}\left\langle\overrightarrow{u z}, \overrightarrow{y_{n}} \vec{z}\right\rangle & =\limsup _{n \rightarrow \infty}\left(\left\langle\overrightarrow{u z}, \overrightarrow{y_{n} x_{n}}\right\rangle+\left\langle\overrightarrow{u z}, \overrightarrow{x_{n}} \vec{z}\right\rangle\right) \\
& \leq \limsup _{n \rightarrow \infty}\left(d(u, z) d\left(y_{n}, x_{n}\right)+\left\langle\overrightarrow{u z}, \overrightarrow{x_{n}} \vec{z}\right\rangle\right) \leq 0 .
\end{aligned}
$$

Using inequality (3.14) and condition $C 1$, we get

$$
\limsup _{n \rightarrow \infty}\left(\delta_{n} d(z, u)+2\left(1-\delta_{n}\right)\left\langle\overrightarrow{u z}, \overrightarrow{y_{n}} \vec{z}\right\rangle\right) \leq 0 .
$$

From (3.2) and Lemma 2.3, we get

$$
d^{2}\left(z, x_{n+1}\right) \leq \delta_{n}^{2} d^{2}(z, u)+\left(1-\delta_{n}\right)^{2} d^{2}\left(z, y_{n}\right)+2 \delta_{n}\left(1-\delta_{n}\right)\left\langle\overrightarrow{u z}, \overrightarrow{y_{n}} \vec{z}\right\rangle
$$

Thus,

$$
d^{2}\left(z, x_{n+1}\right) \leq\left(1-\delta_{n}\right) d^{2}\left(z, x_{n}\right)+\delta_{n}\left(\delta_{n} d^{2}(z, u)+2\left(1-\delta_{n}\right)\left\langle\overrightarrow{u z}, \overrightarrow{y_{n} z}\right\rangle\right) .
$$

By Lemma 2.9, inequalities (3.15) and (3.16), we obtain that $x_{n} \rightarrow z$ as $n \rightarrow \infty$.

Case 2. Assume sequence $\left\{d\left(z, x_{n}\right)\right\}$ is not non-increasing. Putting $\left\{a_{n}\right\}=\left\{d^{2}\left(z, x_{n}\right)\right\}$, we find that there exists a subsequence $\left\{n_{i}\right\}$ of $\{n\}$ such that, for all $i \in \mathbb{N}, a_{n_{i}} \leq a_{n_{i}+1}$. For some sufficiently large $N$ and for all $n \geq N$, we define a map $\tau: \mathbb{N} \rightarrow \mathbb{N}$ by

$$
\tau(n)=\max \left\{k \leq n: a_{k} \leq a_{k+1}\right\} .
$$

Then, it follows from Lemma 2.10 that $\tau(n)$ is non-decreasing with $\tau(n) \rightarrow \infty$ as $n \rightarrow \infty$ and $a_{\tau(n)} \leq$ $a_{\tau(n)+1}, a_{n} \leq a_{\tau(n)+1}$. Using the fact that $\delta_{\tau(n)} \rightarrow 0$ as $\tau(n) \rightarrow \infty$, equation (3.6) and (3.7), we obtain $d\left(x_{\tau(n)}, T x_{\tau(n)}\right) \rightarrow 0, d\left(x_{\tau(n)}, T^{2} x_{\tau(n)}\right) \rightarrow 0$ as $\tau(n) \rightarrow 0$. Following similar argument as in Case 1 , we see from (3.15) that

$$
\limsup _{n \rightarrow \infty}\left(\delta_{\tau(n)} d(z, u)+2\left(1-\delta_{\tau(n)}\right)\left\langle\overrightarrow{u z}, \overrightarrow{y_{\tau(n)}}\right\rangle\right) \leq 0
$$


It follows from equation (3.16) that

$$
a_{\tau(n)+1} \leq a_{\tau(n)}+\delta_{\tau(n)}\left[\delta_{\tau(n)} d^{2}(z, u)+2\left(1-\delta_{\tau(n)}\right)\left\langle\overrightarrow{u z}, \overrightarrow{y_{n}} \vec{z}\right\rangle-a_{\tau(n)}\right] .
$$

From the fact that $a_{\tau(n)} \leq a_{\tau(n)+1}$ and $\delta_{\tau(n)}>0$, the above inequalities give

$$
a_{\tau(n)} \leq \delta_{\tau(n)} d^{2}(z, u)+2(1-\delta)\left\langle\overrightarrow{u z}, \overrightarrow{y_{n} z}\right\rangle
$$

Thus, using (3.17) we get

$$
\lim _{\tau(n) \rightarrow \infty} a_{\tau(n)}=\lim _{\tau(n) \rightarrow \infty} a_{\tau(n)+1}=0 .
$$

Since $0 \leq a_{n} \leq a_{\tau(n)+1}$, this implies that $\lim _{n \rightarrow \infty} a_{n}=\lim _{n \rightarrow \infty} d\left(z, x_{n}\right)=0$. Therefore, $x_{n} \rightarrow z$ as $n \rightarrow \infty$. Hence in view of the above two cases, we see that $\left\{x_{n}\right\}$ converges strongly to $z=\mathrm{P}_{A(T)}(u) \in A(T)$. This completes the proof.

In view of Remark 3.1, the following results can be obtained by applying Theorem 3.1.

Corollary 3.1. Let $C$ be a nonempty, convex subset of a complete $C A T(0)$ space $X$ which satisfy property $(\mathbb{S})$ and condition $\left(\bar{Q}_{4}\right)$. Let $T: C \rightarrow C$ be a further generalized hybrid mapping such that $A(T) \neq \emptyset$. Let $\left\{x_{n}\right\}$ be a sequence defined by (3.2). Then $\left\{x_{n}\right\}$ converges strongly to $z=P_{A(T)}(u)$.

Proof. Since a further 2-generalized hybrid is reduced to a further generalized hybrid mapping if $\alpha_{1}=$ $\beta_{1}=\varepsilon_{2}=0$. It follows from Theorem 3.1 that sequence $\left\{x_{n}\right\}$ converges strongly to $z=\mathrm{P}_{A(T)}(u)$. This completes the proof.

Corollary 3.2. Let $C$ be a nonempty, convex subset of a complete $C A T(0)$ space $X$ which satisfy property $(\mathbb{S})$ and condition $\left(\bar{Q}_{4}\right)$. Let $T: C \rightarrow C$ be a normally 2-generalized hybrid mapping such that $A(T) \neq \emptyset$. Let $\left\{x_{n}\right\}$ be a sequence defined by (3.2). Then $\left\{x_{n}\right\}$ converges strongly to $z=P_{A(T)}(u)$.

Proof. If $\varepsilon_{1}=\varepsilon_{2}=0$, then a further 2-generalized hybrid is reduced to a further generalized hybrid mappings. From Theorem 3.1 we see that $\left\{x_{n}\right\}$ converges strongly to $z=\mathrm{P}_{A(T)}(u)$. This completes the proof.

\section{REFERENCES}

[1] I.D. Berg, I.G. Nicolaev, Quasilinearization and curvature of Alexandrov spaces, Geom. Dedicata 133 (2008), $195-218$.

[2] J.B. Baillon, Un theoreme de type ergodique pour less contractions nonlinears dans un espaces de Hilbert, C.R. Acad. Sci. Paris, Ser. A-B 280 (1975), 1511-1541.

[3] M. Bridson, A. Haefliger, A Metric Space of Non-Positive Curvature, Springer, Berlin, (1999).

[4] C.E. Chidume, A.U. Bello, P. Ndambomve, Strong and $\Delta$-convergence theorems for common fixed points of a finite family of multivalued demicontractive mappings in CAT(0) spaces, Abstr. Appl. Anal. 2014 (2014), 805168.

[5] A. Cuntavepanit, W. Phuengrattana, Iterative approximation of attractive points of further generalized hybrid mappings in Hadamard spaces, Fixed Point Theory Appl. 2019 (2019), 3.

[6] H. Dehghan, J. Rooin, Metric projection and convergence theorems for nonexpansive mappings in Hadamard spaces, arXiv:1410.1137VI [math.FA], 5 Oct., 2014.

[7] S. Dhompongsa, B. Panyanak, On $\Delta$-convergence theorems in CAT(0) spaces, Comput. Math. Appl. 56 (2008), $2572-$ 2579.

[8] S. Dhompongsa, A. Kaewkhao, B. Panyanak, On Kirk's strong convergence theorems for multivalued nonexpansive mappings on CAT(0) spaces, Nonlinear Anal. 75 (2012), 459-468.

[9] K. Goebel, S. Reich, Uniform Convexity, Hyperbolic Geometry and Nonexpansive Mappings, Marcel Dekker, New York, (1984). 
[10] A. Kaewkhao, W. Inthakon, K. Kunwai, Attractive points and convergence theorems for normally generalized hybrid mappings in CAT(0) spaces, Fixed Point Theory Appl. 2015 (2015), 96.

[11] B.A. Kakavandi, Weak topologies in complte CAT(0) metric spaces, Proc. Amer. Math. Soc. 141 (2013), $1029-1039$.

[12] B.A. Kakavandi, M. Amini, Duality and subdifferential for convex functions on complete CAT(0) metric spaces, Nonlinear Anal. 73 (2010), 3450-3455.

[13] W.A. Kirk, B. Panyanak, A concept of convergence in geodesic spaces, Nonlinear Anal. 68 (2008), 3689-3696.

[14] P. Kocourek, W. Takahashi, J.-C. Yao, Fixed points and weak convergence theorems for generalized hybrid mappings in Hilbert spaces, Taiwanese J. Math. 14 (2010), 2497-2511.

[15] F. Kohsaka, W. Takahashi, Fixed points for a class of nonlinear mappings related to a maximal monotone operators in Banach spaces, Arch. Math.(Basel) 19 (2008), 166-177.

[16] F. Kohsaka, W. Takahashi, Existence and approximation of firmly nonexpansive type mappings in Banach spaces, SIAM J. Optim. 19 (2008), 824-835.

[17] A. Kondo, W. Takahashi, Attractive point and weak convergence theorems normally N-generalized hybrid mappings in Hilbert spaces, Linear Nonlinear Anal. 3 (2017), 297-310.

[18] A. Kondo, W. Takahashi, Strong convergence theorems of Halpern's type for normally 2-generalized hybrid mappings in Hilbert spaces, J. Nonlinear Convex Anal. 19 (2018), 617-631.

[19] K. Kunwai, A. Kaewkhao, W. Inthakon, Properties of attractive point in CAT(0) spaces, Thai. J. Math. 13 (2015), $109-121$.

[20] P.-E. Mainge, Strong convergence of projected subgradient methods for nonsmooth and nonstrictly convex minimization, Set-Valued Anal. 16 (2008), 899-912.

[21] S. Reich, I. Shafrir, Nonexpansive iterations in Hyperbolic spaces, Nonlinear Anal. 15 (1990), 537-558.

[22] S.H. Khan, Iterative approximations of common attractive points of further generalized hybrid mappings, Fixed Point Theory Appl. 2018 (2018), 69.

[23] W. Takahashi, Fixed point theorems for new nonlinear mappings in Hilbert spaces, J. Nonlinear Convex Anal. 11 (2010), 79-88.

[24] W. Takahashi, Y. Takeuchi, Nonlinear ergodic theorem without convexity for generalized hybrid mappings in Hilbert spasces, J. Nonlinear Convex Anal. 12 (2011), 399-406.

[25] W. Takahashi, N.-C. Wong, J.-C. Yao, Attractive points and Halpern type strong convergence theorems in Hilbert spaces, J. Fixed point Theory Appl. 17 (2015), 301-311.

[26] W. Takahashi, Nonlinear Functional Analysis-Fixed Point Theory and its Application, Yokohama Publishers, Yokohama, (2000).

[27] W. Takahashi, N.-C. Wong, J.-C. Yao, Attractive point and weak convergence theorem for new generalized hybrid mappings in Hilbert spaces, J. Nonlinear Convex Anal. 13 (2012), 745-757.

[28] H.-K. Xu, Another control condition in an iterative method for nonexpansive mappings, Bull. Aust. Math. Soc. 65 (2002), 109-113. 